\title{
Safety and Disease Evolution of Fixed-Dose Combination of Antitubercular Treatment Compared to Separate-Drugs Preparation in Extra-Pulmonary Tuberculosis
}

\author{
Houda Ben Ayed ${ }^{1,2} \cdot$ Makram Koubaa ${ }^{2,3} \cdot$ Khaoula Rekik $^{2,3} \cdot$ Chakib Marrakchi $^{2,3} \cdot$ Tarak Ben Jemaa $^{2,3}$. \\ Mohamed Makhlouf $^{2,4} \cdot$ Aida Mustapha $^{2,4} \cdot$ Manel Turki $^{5}$ - Sourour Yaich ${ }^{1} \cdot$ Maissa Ben Jemaa $^{1} \cdot$ Imed Maaloul $^{2,3}$. \\ Jamel Damak ${ }^{1} \cdot$ Mounir Ben Jemaa ${ }^{2,3}$
}

Published online: 16 August 2018

(C) Springer Nature Switzerland AG 2018

\begin{abstract}
Purpose of Review Extra-pulmonary tuberculosis (EPTB) treatment was previously based on separate-drugs preparation (SDP). In the hope of reducing the rates of treatment default and resistance, the WHO have recommended the use of fixed-dose combination (FDC) for first-line in EPTB. We aimed to compare the tolerance and the disease evolution between FDC and SDP regimens in EPTB patients.

Recent Findings We conducted a retrospective study including 388 cases of EPTB hospitalized between 1996 and 2016. We compared anti-tuberculosis treatment outcomes and the disease evolution between patients receiving FDC and those receiving SDP. The main EPTB site was lymph node (39.2\%). There were no statistical differences between the two groups in terms of musculoskeletal, hematological disorders, cutaneous events, and hepatotoxicity. We noted that neurological disorder (OR $=12 ; p$ $<0.001$ ), notably paresthesia ( $\mathrm{OR}=16 ; p<0.001)$, and retrobulbar neuritis ( $\mathrm{OR}=10 ; p=0.006)$, as well as gastro-intestinal intolerance $(\mathrm{OR}=4 ; p=0.015)$ including nausea $(\mathrm{OR}=8.9 ; p=0.011)$ and vomiting $(\mathrm{OR}=1.1 ; p=0.005)$ were significantly more frequent in the SDP group. The disease evolution comparison showed that complicated forms were statistically more frequent in the SDP group than those in the FDC group ( $\mathrm{OR}=2.4 ; p=0.003)$, while there was no significant difference in relapse, sequelae, and death frequencies between the two groups.

Summary SDP tolerance was characterized by higher frequency of paresthesia, retrobulbar neuritis, nausea, and vomiting than FDC. Evolutionary profiles were similar, except a higher risk of complicated forms in SDP.
\end{abstract}

Keywords Extra-pulmonary tuberculosis $\cdot$ Evolution $\cdot$ Fixed-dose combination $\cdot$ Safety $\cdot$ Antitubercular

Houda Ben Ayed

drhoudabayed@gmail.com; makram.koubaa@gmail.com

1 Community Health and Epidemiology Department, Hedi Chaker University Hospital, University of Sfax, Sfax, Tunisia

2 Extra-pulmonary Tuberculosis Research Unit, Hedi Chaker University Hospital, University of Sfax, Sfax, Tunisia

3 Infectious Diseases Department, Hedi Chaker University Hospital, University of Sfax, Sfax, Tunisia

4 Basic Health Care Direction, Hedi Chaker University Hospital, University of Sfax, Sfax, Tunisia

5 Department of Pharmacy, Hedi Chaker University Hospital, University of Sfax, Sfax, Tunisia

\section{Introduction}

Tuberculosis (TB) is a global pandemic and a major cause of morbidity and mortality worldwide. Although TB most commonly affects the lungs, other extra-pulmonary organs can get involved. Extra-pulmonary tuberculosis (EPTB) is one of the major causes of death from a curable infectious disease. Recently, there has been a renew interest in EPTB, since it represents an increasing multisystem disease, which may lead to serious outcomes in lack of an adequate treatment [1].

TB control programs targeted to ensure an effective treatment and a rapid and lasting cure. Despite the availability of effective anti-TB drugs, achieving a high cure rate is challenging and critical, for several reasons: poor drug adherence [2], inadequate doses or dosing frequency [3, 4], and drugs given 
in unsupervised manner [5] may lead to treatment failure and then promote drug resistance. Development of multi-drug resistance is a further threat to TB control, especially in TBendemic countries. Pulmonary and EPTB should be treated with the same drugs, and in some cases of EPTB (such as TB meningitis and bone or joint TB), the recommended therapy is longer than the standard TB regimen [6]. Previously, the usual TB treatment regimen was based on separate-drugs preparation (SDP) of isoniazid (INH), rifampin (RIF), pyrazinamide (PZA), and ethambutol (EMB). Otherwise, this classic regimen was associated with poor compliance to treatment and then further risk of drug resistance [7]. In the hope of reducing the rates of treatment default and halting the possible increase in resistance rates, the World Health Organization (WHO) and the International Union Against Tuberculosis and Lung Disease have recommended the use of fixed-dose combination (FDC) for first-line treatment of drug-susceptible TB [8]. In our country, we have adopted the FDC regimens in TB treatment since 2009, for logistic reasons and easier applicability. Despite the availability of effective antimycobacterial treatment, several adverse outcomes are common in patients with EPTB [9]. Little information is available on the tolerance of the FDC and the disease evolution of EPTB in high-burden countries. In light of this, our study aimed to compare the tolerance and the disease evolution between FDC and SDP regimens in patients with EPTB.

\section{Methods}

\section{Study Design}

We performed an observational study using retrospective information from medical records of patients with EPTB, hospitalized within a department of infectious diseases. We included all cases of EPTB hospitalized from January 1996 to December 2016. We compared anti-tuberculosis treatment outcomes and the disease evolution between patients receiving FDC and those receiving SDP.

\section{Eligibility Criteria}

All new EPTB cases weighing $\geq 30 \mathrm{~kg}$ including adults and children registered during the study period were included. Only EPTB patients who received either FDC or SDP for the entire duration of treatment and who were adherent to the treatment protocol were eligible for the analysis. We included all EPTB cases during the study period. The diagnosis was confirmed based on histological findings or the presence of a "positive microbiology," including a positive culture or molecular biology result in the infection site sample. In default, the diagnosis was based on strong clinical, radiological, and evolutionary evidence consistent with active extrapulmonary disease.

We excluded all EPTB who had a history of a recurrent tuberculosis, a drug-induced hepatitis, suspected or known cases of acute and chronic liver disease regardless of their origin, renal failure, peripheral optic neuritis, and psychiatric illness. Patients with a history of gout and any condition that might prove fatal during the study period (e.g., metastatic cancer), poor general condition requiring additional measures to ensure survival and patients receiving immunosuppressive treatment (e.g., corticosteroids) were also ruled out.

\section{Anti-tuberculosis Treatment Regimens}

EPTB patients were treated according to the WHO guidelines: standard first-line treatment consists of 2 months of daily RIF, INH, PZA, and EMB, followed by at least 4 months of daily INH and RIF, depending on the involved site. Treatment was administered daily by the nurse early morning on empty stomach.

Choice of the regimen was based on the introduction of the FDC in 2009, when physicians started to prescribe FDC for most EPTB patients. When treatment was provided as SDP, the following were available as follows: INH $100 \mathrm{mg}$, RIF $300 \mathrm{mg}$, PZA $500 \mathrm{mg}$, and EMB $400 \mathrm{mg}$. As to FDC, they were administrated in tablets as follows: 2-month RHZE during the intensive phase (RIF $150 \mathrm{mg} / \mathrm{INH} 75 \mathrm{mg} / \mathrm{PZA}$ $400 \mathrm{mg} / \mathrm{EMB} 275 \mathrm{mg}$ ) and 4-month RH for the continuation phase (RIF $150 \mathrm{mg} / \mathrm{INH} 75 \mathrm{mg}$ ).

Dosages were calculated based on the patient's weight to determine how much of each preparation were required. The daily dosage of FDC consisted of two tablets for patients weighing 30-39 kg, three tablets for patients weighing 40 $54 \mathrm{~kg}$, four tablets for patients weighing 55-69 kg, and five tablets for patients weighing $70 \mathrm{~kg}$ or more.

\section{Data Collection}

We retrospectively collected data from medical records, laboratory, and pharmacological databases including patients' demographics, co-morbidities, clinical features, and laboratory parameters at the time of the diagnosis and during the whole period of treatment. These include age, gender, conventional risk factors, co-morbidities, symptoms, and the treatment regimen. We recorded the main clinical and biological adverse events during the follow-up of EPTB patients.

\section{Adverse Events and Disease Evolution Assessments}

Adverse events, including symptoms and altered laboratory results, were closely assessed during the follow-up of EPTB patients. These include hepatotoxicity (defined as functional 
disturbance (aspartate aminotransferase (AST) or alanine aminotransferase $($ ALT) $) \geq 3$ upper limit normal, cholestasis), cutaneous adverse events (skin rash, toxidermia, and urticaria), gastro-intestinal adverse events (nausea, vomiting, or epigastric and abdominal pain), and neurological disorder (paresthesia, convulsion, retrobulbar neuritis, nervousness, and behavioral disorder). We also recorded musculoskeletal adverse events (myalgia and arthralgia) and hematological disorder (thrombocytopenia and leucopenia). We compared the incidence of these events between the two treatment regimens receiving either FDC or SDP.

The disease evolution was assessed by the frequency of relapse, complicated forms, sequelae, and death during the follow-up.

\section{Statistical Analysis}

Statistical analysis was performed using SPSS20. The results of quantitative variables were presented as mean \pm standard deviation (SD) or median and interquartile range (IQR), those of qualitative variables as percentages. When the variables distribution was normal, we used the $t$ test to compare two means. For categorical variables, we used the Chi-square test in independent samples. When the variables were not normally distributed, we used non-parametric tests such as Wilcoxon, Mann-Whitney, and Kruskal-Wallis tests when appropriate. $p$ values lower than 0.05 were considered statistically significant.
Table 1 Characteristics of the study population

\begin{tabular}{|c|c|c|}
\hline \multicolumn{3}{|l|}{ Variables } \\
\hline $\operatorname{Total}\left(N^{\mathrm{b}}, \%\right)$ & 388 & 100 \\
\hline Age (mean; $\mathrm{SD}^{\mathrm{c}}$ ) (years) & 40.8 & 18 \\
\hline Gender (females; $N^{\mathrm{b}}, \%$ ) & 243 & 62.6 \\
\hline Rural area $\left(N^{\mathrm{b}}, \%\right)$ & 258 & 66.4 \\
\hline Lifestyle $\left(N^{\mathrm{b}}, \%\right)$ & 58 & 14.2 \\
\hline Smoking & 40 & 10.3 \\
\hline Alcohol consumption & 11 & 2.8 \\
\hline \multicolumn{3}{|l|}{ Clinical signs $\left(N^{\mathrm{b}}, \%\right)$} \\
\hline Fever & 256 & 66 \\
\hline Asthenia & 247 & 63.7 \\
\hline Anorexia & 206 & 53 \\
\hline Severe weight loss & 156 & 40.2 \\
\hline Night sweats & 127 & 32.8 \\
\hline \multicolumn{3}{|l|}{$\mathrm{EPTB}^{\mathrm{a}}$ diagnosis $\left(N^{\mathrm{b}}, \%\right)$} \\
\hline Histopathological findings & 228 & 59 \\
\hline Strong clinical, radiological, and evolutionary arguments & 126 & 32. \\
\hline Positive culture specimen & 48 & 12.4 \\
\hline PCR-positive sample & 10 & 2.6 \\
\hline \multicolumn{3}{|l|}{$\operatorname{EPTB}^{\mathrm{a}}$ sites $\left(N^{\mathrm{b}}, \%\right)$} \\
\hline Lymph node & 152 & 39.2 \\
\hline Bone and joint & 63 & 16.2 \\
\hline Neuro-meningeal & 63 & 16.2 \\
\hline Abdominal & 61 & 15.7 \\
\hline Urogenital & 53 & 13.7 \\
\hline Cutaneous & 14 & 3.6 \\
\hline Pleural & 8 & 2.1 \\
\hline \multicolumn{3}{|l|}{ Treatment regimen } \\
\hline $\operatorname{SDP}\left(N^{\mathrm{b}}, \%\right)$ & 259 & 66.8 \\
\hline $\operatorname{FDC}\left(N^{\mathrm{b}}, \%\right)$ & 129 & 33.2 \\
\hline Treatment duration (months) (mean; $\mathrm{SD}^{\mathrm{c}}$ ) & 12.4 & 4.9 \\
\hline
\end{tabular}




\section{Results}

\section{Patients' Characteristics}

Totally, 388 EPTB patients who fulfilled the eligibility criteria were included in the analysis, which represented $86.8 \%$ of the total EPTB caseload registered during the study period. There were 243 females $(62.6 \%)$. The sex ratio (male/female) was 0.6. The mean age was $40.8 \pm 18$ years. We found 258 cases coming from a rural area $(66.4 \%)$. There were 40 smokers $(10.3 \%)$. The main symptoms were fever in 256 cases $(66 \%)$ and asthenia in 247 cases $(63.7 \%)$. The diagnosis was mainly based on histological findings in 228 cases (59\%). There were 48 positive culture specimens (12.4\%) and 10 PCR-positive samples $(2.6 \%)$. In the remaining cases, the diagnosis of EPTB was made by strong clinical, radiological, and evolutionary arguments (126 cases; 32.5\%). The main EPTB sites were lymph node in 152 cases (39.2\%) followed by neuromeningeal and bone and joint sites in 63 cases (16.2\%), each. FDC drugs were administered in 129 cases $(33.2 \%)$. The mean duration of the anti-tuberculous treatment was $12.4 \pm$ 4.9 months (Table 1).

Demographic characteristics, co-morbidities and clinical features were similar between the two groups, with no significant difference. The mean doses ranged from 3.8 to $5.25 \mathrm{mg} /$ $\mathrm{kg}$ for INH, 8.5 to $12.1 \mathrm{mg} / \mathrm{kg}$ for RIF, 21.9 to $30 \mathrm{mg} / \mathrm{kg}$ for $\mathrm{PZA}$, and 15 to $20.7 \mathrm{mg} / \mathrm{kg}$ for EMB. The daily doses by body weight categories for FDP and SDP are shown in Table 2.

\section{Safety Assessment}

The main adverse events were hepatotoxicity and neurological disorders in $14.4 \%$ and $11.9 \%$, respectively. Subgroup comparison of adverse events showed that there was no statistical difference between the two groups in terms of musculoskeletal, hematological disorders, cutaneous events, and hepatotoxicity. Otherwise, we noted that neurological disorder (odds ratio $(\mathrm{OR})=12 ; p<0.001)$, notably paresthesia $(\mathrm{OR}=$ $16 ; p<0.001)$, and retrobulbar neuritis $(\mathrm{OR}=10 ; p=0.006)$ as well as gastro-intestinal intolerance $(\mathrm{OR}=4 ; p=0.015)$ including nausea $(\mathrm{OR}=8.9 ; p=0.011)$ and vomiting $(\mathrm{OR}=1.1$; $p=0.005$ ) were significantly more frequent in the SDP group (Table 3).

\section{Disease Evolution}

The evolution of the disease was marked by the occurrence of complicated forms in 86 cases (22\%) and sequelae in 60 cases (15.4\%). We noted that complicated forms were statistically more frequent in the SDP group than those in the FDC group $(\mathrm{OR}=2.4 ; p=0.003)$, while there was no significant difference in relapse, sequelae, and death frequencies between the two groups (Table 4).

\section{Discussion}

In the present study, we assessed the treatment response and the disease evolution during the follow-up in patients with EPTB. FDC use was a safe and easily administered treatment in a long-course treatment regimen. In fact, it has been reported that FDC improve medication compliance by $24-26 \%$ in patients with chronic illness, compared with free-drug combination regimen [10].

Several previous studies including randomized clinical trials and prospective cohorts showed no significant differences in terms of safety and patient adherence to treatment $[2,11]$. A meta-analysis including 13 randomized clinical trials reported that there was no significant difference in terms of either serious adverse events or adverse events that led to discontinuation of therapy [6]. Similarly, Lienhardt C. showed that adverse events related to trial drugs were similarly distributed
Table 2 Daily doses by body weight category for fixed-dose combinations and single-drug preparations of antitubercular treatment

\begin{tabular}{|c|c|c|c|c|c|c|c|c|}
\hline \multirow[t]{2}{*}{ Patients' weight category (kg) } & \multicolumn{4}{|c|}{$\mathrm{FDC}^{\mathrm{a}}$ dose (mean, $\left.\mathrm{mg} / \mathrm{kg}\right)$} & \multicolumn{4}{|c|}{$\mathrm{SDP}^{\mathrm{b}}$ dose (mean, $\left.\mathrm{mg} / \mathrm{kg}\right)$} \\
\hline & $\mathrm{INH}^{\mathrm{c}}$ & $\mathrm{RIF}^{\mathrm{d}}$ & $\mathrm{PZA}^{\mathrm{e}}$ & $\mathrm{EMB}^{\mathrm{f}}$ & INH & RIF & PZA & EMB \\
\hline Total & 4.6 & 9.3 & 23.8 & 16.8 & 4.3 & 10.8 & 27.3 & 19.5 \\
\hline $30-39$ & 5 & 9.5 & 26.3 & 18 & 5.25 & 11.6 & 30 & 20.7 \\
\hline $40-54$ & 4.9 & 10.1 & 25.3 & 18.5 & 4.4 & 12.1 & 28.6 & 20 \\
\hline $55-70$ & 4.6 & 9.4 & 23.9 & 17.1 & 4.2 & 10.2 & 27.3 & 19.7 \\
\hline$>70$ & 4.2 & 8.5 & 21.9 & 15 & 3.8 & 9.3 & 23.8 & 17.5 \\
\hline \multicolumn{9}{|l|}{${ }^{\mathrm{a}} F D C$, fixed-dose combination } \\
\hline \multicolumn{9}{|l|}{${ }^{\mathrm{b}} S D P$, separate-drugs preparation } \\
\hline \multicolumn{9}{|l|}{${ }^{\mathrm{c}} I N H$, isoniazid } \\
\hline \multicolumn{9}{|l|}{${ }^{\mathrm{d}} R I F$, rifampicin } \\
\hline \multicolumn{9}{|l|}{${ }^{\mathrm{e}} P Z A$, pyrazinamide } \\
\hline${ }^{\mathrm{f}} E M B$, ethambutol & & & & & & & & \\
\hline
\end{tabular}


Table 3 Comparison of adverse events of antitubercular treatment between fixed-dose combination and separate-drugs preparation in patients with extra-pulmonary tuberculosis

\begin{tabular}{|c|c|c|c|c|c|}
\hline \multirow[t]{2}{*}{ Variables } & \multicolumn{3}{|c|}{ Treatment regimens $\left(N^{\mathrm{a}}, \%\right)$} & \multirow[t]{2}{*}{$\mathrm{OR}^{\mathrm{d}}$} & \multirow[t]{2}{*}{$p$} \\
\hline & Total $(n=388)$ & $\operatorname{FDC}^{\mathrm{b}}(n=129)$ & $\operatorname{SDP}^{\mathrm{c}}(n=259)$ & & \\
\hline Neurological disorder & $46(11.9)$ & $2(1.6)$ & $44(17)$ & 12 & $<0.001$ \\
\hline Paresthesia & $30(7.7)$ & $1(0.8)$ & $29(11.2)$ & 16 & $<0.001$ \\
\hline Convulsion & $2(0.5)$ & $0(0)$ & $2(0.8)$ & 1 & 0.3 \\
\hline Nervousness & $8(2.1)$ & $0(0)$ & $8(3.1)$ & 1.03 & 0.056 \\
\hline Retrobulbar neuritis & $20(5.2)$ & $1(0.8)$ & $19(7.3)$ & 10 & 0.006 \\
\hline Behavioral disorders & $5(1.3)$ & $0(0)$ & $5(1.9)$ & 1 & 0.1 \\
\hline Gastro-intestinal intolerance & $26(6.7)$ & $3(2.3)$ & $23(8.9)$ & 4 & 0.015 \\
\hline Epigastric and abdominal pain & $20(5.2)$ & $3(2.3)$ & $17(6.6)$ & 2.9 & 0.075 \\
\hline Nausea & $18(4.6)$ & $1(0.8)$ & $17(6.6)$ & 8.9 & 0.011 \\
\hline Vomiting & $15(3.9)$ & $0(0)$ & $15(5.8)$ & 1.1 & 0.005 \\
\hline Musculoskeletal disorder & $3(0.8)$ & $1(0.8)$ & $2(0.8)$ & 1 & 0.9 \\
\hline Myalgia & $3(0.8)$ & $1(0.8)$ & $2(0.8)$ & 1 & 0.9 \\
\hline Arthralgia & $2(0.5)$ & $0(0)$ & $2(0.8)$ & 1 & 0.4 \\
\hline Hematological disorder & $10(2.6)$ & $3(2.3)$ & $7(2.7)$ & 1.1 & 0.5 \\
\hline Leucopenia & $10(2.6)$ & $3(2.3)$ & $7(2.7)$ & 1.1 & 1 \\
\hline Thrombocytopenia & $2(0.5)$ & $0(0)$ & $2(0.8)$ & 1 & 0.6 \\
\hline Hepatotoxicity & $56(14.4)$ & $18(14)$ & $38(14.7)$ & 1 & 0.8 \\
\hline Cytolysis & $54(13.9)$ & $18(14)$ & $36(13.9)$ & 1 & 0.9 \\
\hline Cholestasis & $7(1.8)$ & $2(1.6)$ & $5(1.9)$ & 1.2 & 0.8 \\
\hline Cutaneous events & $27(7)$ & $11(8.5)$ & $16(6.2)$ & 1.42 & 0.4 \\
\hline Toxidermia & $6(1.5)$ & $2(1.6)$ & $4(1.5)$ & 1 & 1 \\
\hline Skin rash & $16(4.1)$ & $6(4.7)$ & $10(3.9)$ & 0.8 & 0.7 \\
\hline Urticaria & $4(1)$ & $2(1.6)$ & $2(0.77)$ & 0.5 & 0.47 \\
\hline \multicolumn{6}{|l|}{${ }^{\mathrm{a}} N$, number } \\
\hline \multicolumn{6}{|l|}{${ }^{\mathrm{b}} F D C$, fixed-dose combination } \\
\hline${ }^{\mathrm{c}} S D P$, separate-drugs preparation & & & & & \\
\hline${ }^{\mathrm{d}} O R$, odds ratio & & & & & \\
\hline
\end{tabular}

vs $0 \% ; p=0.03$ ) in patients with pulmonary TB [13]. Previous studies have shown that the use of multi-drug regimens can cause undesirable adverse drug reactions of varying degrees of severity, such as neurological disorders [14]. Retrobulbar optic neuritis is the main adverse event associated with EMB. Possible explanation for this is that the EMB dose used in the SDP regimen was a weight-based dose estimated around
Table 4 Comparison of the disease evolution between fixeddose combination and separatedrugs preparation of antitubercular treatment in patients with extra-pulmonary tuberculosis

\begin{tabular}{lccccc}
\hline Disease evolution & Total $\left(N^{\mathrm{a}}, \%\right)$ & $\operatorname{FDC}^{\mathrm{b}}(N, \%)$ & $\operatorname{SDP}^{\mathrm{c}}(N, \%)$ & $\mathrm{OR}^{\mathrm{d}}$ & $p$ \\
\hline Total & $388(100)$ & $129(100)$ & $259(100)$ & & \\
Complicated forms & $86(22)$ & $17(13.2)$ & $69(26.6)$ & 2.4 & 0.003 \\
Relapse & $21(5.4)$ & $3(2.3)$ & $18(6.9)$ & 3.1 & 0.05 \\
Sequelae & $60(15.4)$ & $16(12.8)$ & $44(17.1)$ & 1.4 & 0.2 \\
Death & $3(0.8)$ & $0(0)$ & $3(1.2)$ & 1 & 0.2 \\
\hline
\end{tabular}

${ }^{\mathrm{a}} N$, number

${ }^{\mathrm{b}} F D C$, fixed-dose combination

${ }^{\mathrm{c}} S D P$, separate-drugs preparation

d $O R$, odds ratio 
$19.5 \mathrm{mg} / \mathrm{kg}$, while in the FDC regimen, patients received a $16.8 \mathrm{mg} / \mathrm{kg}$ dose of EMB. In this context, previous studies reported that ocular toxicity was the most important frequent adverse event and was correlated with the frequency of exposures [15]. Two important points must be surmised: first, accurate dosing according to patient's weight is an important aspect of prescribing in order to maximize antibiotic therapy and to minimize side effects. Then, physicians should reexamine the weight bands of patients receiving a longcourse treatment and adjust the drugs dosage accordingly.

Additionally, in a systematic review evaluating the tolerance of TB treatment regimens, it has been reported that there was a statistical difference between the two regimens in the occurrence of gastro-intestinal adverse effects [16]. On the other hand, previous studies showed that SDP caused more digestive intolerance such as nausea and vomiting compared with FDC, which was consistent with our study [17-19]. Haroon M. found that $39 \%$ of patients undergoing an antiTB treatment could not continue the treatment course due to either gastro-intestinal intolerance or hypertransaminesemia [20].

In terms of dermatologic, hepatic and musculoskeletal disorders, there was no significant difference between FDC and SDP [12]. Likewise, Gallardo CR. reported that there were no differences in adverse events leading to discontinuation of therapy [6]. These findings are consistent with our results.

In contrast with pulmonary $\mathrm{TB}$, a cure definition is challenging in EPTB patients, since bacteriological evaluation of the response to treatment is often limited by the difficulty in obtaining follow-up specimens. Treatment response often must be judged on the basis of clinical and radiographic findings [9]. In this study, we assessed the unfavorable outcomes among treated patients based on relapse rate and the frequency of complicated forms, sequelae, and deaths. We noted that there were no significant differences in terms of disease evolution between the two regimens, except complicated forms. A meta-analysis comparing FDC and SDP regimens in pulmonary TB patients showed that there was no significant difference between the two groups for all causes of death, but a significant trend towards a higher number of relapses with FDC was found [6]. It is worthy to note that EPTB evolution must be interpreted according to the sites involved, the severity of the disease, and the patient's immune status.

This is an original study evaluating the treatment safety and the disease evolution in EPTB patients. However, it had several limitations. With the retrospective cohort design, assessment of potential adverse events remains perplexing, since the treatment was administered over different periods. Besides, the sample size was only sufficient to illustrate the different treatment adverse events, but remain insufficient to compare accurately the two treatment regimens.

\section{Conclusions}

In conclusion, our study highlighted the tolerance and the disease evolution of FDC compared to SDP regimen. Except some neurological and gastro-intestinal disorders in the SDP group, a similar safety profile was illustrated between the FDC and SDP regimens. This finding is reassuring since there are other potential advantages associated with FDC use. Introducing FDC for the treatment of EPTB seems to be a promising solution to improve the treatment acceptability by the patients. Further prospective randomized studies or clinical trials with larger number of patients are warranted in order to confirm these findings.

\section{Compliance with Ethical Standards}

Conflict of Interest No potential conflict of interest relevant to this article was reported.

Human and Animal Rights and Informed Consent This article does not contain any studies with human or animal subjects performed by any of the authors.

Abbreviations $T B$, tuberculosis; $E P T B$, extra-pulmonary tuberculosis; $S D P$, separate-drugs preparation; $I N H$, isoniazid; RIF, rifampin; $P Z A$, pyrazinamide; $E M B$, ethambutol; $W H O$, World Health Organization; $F D C$, fixed-dose combination; $A S T$, aspartate aminotransferase; $A L T$, alanine aminotransferase; $S D$, standard deviation; $I Q R$, interquartile range; $O R$, odds ratio

\section{References}

1. Kulchavenya E. Extrapulmonary tuberculosis: are statistical reports accurate? Ther Adv Infect Dis. 2014;2:61-70.

2. Wu J-T, Chiu C-T, Wei Y-F, Lai Y-F. Comparison of the safety and efficacy of a fixed-dose combination regimen and separate formulations for pulmonary tuberculosis treatment. Clinics. 2015;70: 429-34.

3. Malangu N, Mngomezulu M. Evaluation of tuberculosis infection control measures implemented at primary health care facilities in Kwazulu-Natal province of South Africa. BMC Infect Dis. 2015;15:117.

4. Wright A, Zignol M, Van Deun A, et al. Epidemiology of antituberculosis drug resistance 2002-07: an updated analysis of the Global Project on Anti-Tuberculosis Drug Resistance Surveillance. Lancet. 2009;373:1861-73.

5. Mitchison DA. How drug resistance emerges as a result of poor compliance during short course chemotherapy for tuberculosis. Int J Tuberc Lung Dis. 1998;2:10-5.

6. Gallardo CR, Rigau Comas D, Valderrama Rodríguez A, et al. Fixed-dose combinations of drugs versus single drug formulations for treating pulmonary tuberculosis. Cochrane Database Syst Rev. 2016;17:CD009913.

7. Monedero I, Caminero J. Evidence for promoting fixed-dose combination drugs in tuberculosis treatment and control: a review. Int $\mathrm{J}$ Tuberc Lung Dis. 2011;15:433-9.

8. Blomberg B, Spinaci S, Fourie B, Laing R. The rationale for recommending fixed-dose combination tablets for treatment of tuberculosis. Bull World Health Organ. 2001;79:61-8. 
9. Lee JY. Diagnosis and treatment of extrapulmonary tuberculosis. Tuberc Respir Dis (Seoul). 2015;78:47-55.

10. Bangalore S, Kamalakkannan G, Parkar S, Messerli FH. Fixeddose combinations improve medication compliance: a meta-analysis. Am J Med. 2007;120:713-9.

11. Aseffa A, Chukwu JN, Vahedi M, Aguwa EN, Bedru A, Mebrahtu T, et al. Efficacy and safety of 'fixed dose' versus 'loose' drug regimens for treatment of pulmonary tuberculosis in two high TBburden African countries: a randomized controlled trial. PLoS One. 2016;11:e0157434.

12. Lienhardt C, Cook SV, Burgos M, Yorke-Edwards V, Rigouts L, Anyo G, et al. Efficacy and safety of a 4-drug fixed-dose combination regimen compared with separate drugs for treatment of pulmonary tuberculosis: the study $\mathrm{C}$ randomized controlled trial. JAMA. 2011;305:1415-23.

13. Al-Shaer MH, Mansour H, Elewa H, Salameh P, Iqbal F. Treatment outcomes of fixed-dose combination versus separate tablet regimens in pulmonary tuberculosis patients with or without diabetes in Qatar. BMC Infect Dis. 2017;17:118.

14. Lv X, Tang S, Xia Y, Wang X, Yuan Y, Hu D, et al. Adverse reactions due to directly observed treatment strategy therapy in Chinese tuberculosis patients: a prospective study. PLoS One. 2013;8:e65037.
15. Griffith DE, Brown-Elliott BA, Shepherd S, McLarty J, Griffith L, Wallace RJ Jr. Ethambutol ocular toxicity in treatment regimens for Mycobacterium avium complex lung disease. Am J Respir Crit Care Med. 2005;172:250-3.

16. Lima GC, Silva EV, Magalhães PO, Naves JS. Efficacy and safety of a four-drug fixed-dose combination regimen versus separate drugs for treatment of pulmonary tuberculosis: a systematic review and meta-analysis. Braz J Microbiol. 2017;48(2):198-207.

17. Gravendeel JM, Asapa AS, Becx-Bleumink M, Vrakking HA. Preliminary results of an operational field study to compare sideeffects, complaints and treatment results of a single-drug shortcourse regimen with a four-drug fixed-dose combination (4FDC) regimen in South Sulawesi, Republic of Indonesia. Tuberculosis. 2003;83:183-6.

18. Bartacek A, Schütt D, Panosch B, Borek M, Group R-FS. Comparison of a four-drug fixed-dose combination regimen with a single tablet regimen in smear-positive pulmonary tuberculosis. Int J Tuberc Lung Dis. 2009;13:760-6.

19. Toujani S, Ben SB, Ben SN, et al. Contribution of fixed-dose combinations in the treatment of tuberculosis. Tunis Med. 2016;94: 401-5.

20. Haroon M, Martin U, Devlin J. High incidence of intolerance to tuberculosis chemoprophylaxis. Rheumatol Int. 2012;32:33-7. 